\title{
MicroRNA-139 suppressed tumor cell proliferation, migration and invasion by directly targeting HDGF in epithelial ovarian cancer
}

\author{
JUNLI LIU, SHUANGLING JIN and RUI WANG \\ Department of Gynaecology and Obstetrics, Changzhi Peace Hospital Affiliated to Changzhi Medical College, \\ Changzhi, Shanxi 046000, P.R. China
}

Received May 30, 2016; Accepted April 5, 2017

DOI: $10.3892 / \mathrm{mmr} .2017 .6956$

\begin{abstract}
The current study investigated the expression and functional roles of microRNA-139 (miR-139) on human epithelial ovarian cancer (EOC). Reverse transcription-quantitative polymerase chain reaction (RT-qPCR) was performed to measure miR-139 expression in EOC tissues and cell lines. The effects of miR-139 on EOC cell proliferation, migration and invasion were assessed using MTT, cell migration and invasion assays, respectively. Subsequently, the molecular mechanism underlying the tumor suppressive roles of miR-139 on EOC was determined by bioinformatics analysis, RT-qPCR, western blotting and the luciferase reporter assay. According to the results, it was identified that miR-139 was significantly downregulated in EOC tissues and cell lines. In addition, restoration of miR-139 suppressed tumor cell proliferation, migration and invasion in EOC. Furthermore, hepatoma-derived growth factor (HDGF) was identified as a target of miR-139 in EOC. Upregulation of HDGF could rescue the inhibitory effects exerted by miR-139 overexpression on EOC cell proliferation, migration and invasion. Collectively, the results indicated that miR-139 was downregulated in EOC, and acted as a tumor suppressor by directly targeting HDGF. To the best of our knowledge, this was the first study to identify that miR-139 contributes to the growth and metastasis of EOC.
\end{abstract}

\section{Introduction}

Ovarian cancer is the second the most common gynecological malignancy, and is the seventh most common cancer in women worldwide, with 239,000 new cases diagnosed in 2012 (1). Epithelial ovarian cancer (EOC) is the major pathological type of ovarian cancer, and accounts for approximately $90 \%$ cases,

Correspondence to: Professor Junli Liu, Department of Gynaecology and Obstetrics, Changzhi Peace Hospital Affiliated to Changzhi Medical College, 110 Yanan South Road, Changzhi, Shanxi 046000, P.R. China

E-mail: jll_changzhi@163.com

Key words: microRNA-139, HDGF, metastasis, epithelial ovarian cancer including serous adenocarcinoma, endometrial adenocarcinoma and clear cell carcinoma (2). Despite advances in the treatments by combining surgical resection, chemotherapy and radiotherapy, the prognosis for patients with EOC remains unsatisfactory, with a five-year overall survival rate of only $30 \%(3,4)$. The poor prognosis of EOC has been demonstrated to be associated with the occurrence of tumor metastasis, and recurrence (5). Therefore, it is of great clinical significance to elucidate the molecular mechanisms, which contribute to the tumorigenesis and tumor development of EOC, and investigate novel therapeutic targets for this disease.

Previous studies have reported the importance of the regulatory roles of microRNAs (miRNAs) in the carcinogenesis and progression of EOC (6-8). miRNAs are a major group of endogenous, non-protein-coding and short RNA molecules approximately 22 nucleotides in length (9). At present, more than 1,000 miRNAs have been validated, which only represent $\sim 1 \%$ of the predicted genes in the genome, and are estimated to regulate the expression of more than $60 \%$ of protein-coding genes $(10,11)$. miRNAs negatively modulate their target genes through binding to the 3'untranslated region (UTR) of target genes for the post-transcriptional regulation, and therefore to participate in a great deal of biological processes, including cell proliferation, cell cycle, apoptosis, invasion, metastasis, glucose and lipid metabolism and infection and immune responses (12-14). Studies have indicated that the abnormal expression of miRNAs serve significant roles in the occurrence and development of tumors, including bladder cancer (15), renal cell carcinoma (16), colorectal cancer (17), breast cancer (18), hepatocellular carcinoma (19) and EOC (20). Therefore, exploring the expression patterns and roles of miRNAs in EOC may provide potential diagnostic and therapeutic targets for the treatments of EOC.

Thus, the current study demonstrated that the expression level of miR-139 was markedly reduced in EOC, and restoration of miR-139 repressed cellular proliferation, migration and invasion. In addition, it was identified that miR-139 directly targeted and downregulated HDGF through binding to its 3'UTR. Finally, it was demonstrated that HDGF overexpression may rescue the inhibitory effects mediated by miR-139 in EOC.

\section{Materials and methods}

Tissue specimens. The current study was approved by the Ethics Committee of Changzhi Peace Hospital Affiliated to 
Changzhi Medical College (Changzhi, China), and informed consent was also obtained from each patient in accordance with the guidelines of Changzhi Peace Hospital Affiliated to Changzhi Medical College. A total of 22 primary EOC tissues and matched adjacent normal tissues were collected from patients with EOC (age range, 41-71 years; median age, 56) who underwent surgical resection at the Department of Gynaecology and Obstetrics, Changzhi Peace Hospital Affiliated to Changzhi Medical College between June 2014 and October 2015. All tissues were immediately snap-frozen in liquid nitrogen following surgical resection and stored at $-80^{\circ} \mathrm{C}$ in a refrigerator until required for RNA extraction.

Cell lines and oligonucleotides transfection. Four EOC cell lines (OVCAR3, CAOV3, SKOV3 and ES-2) and the human normal ovarian epithelial cell line (NOEC) were obtained from the American Type Culture Collection (ATCC; Manassas, VA, USA). All cell lines were cultured in RPMI-1640 medium or Ham's F-12 medium containing 10\% FBS, $100 \mathrm{mg} / \mathrm{ml}$ penicillin and $100 \mathrm{mg} / \mathrm{ml}$ streptomycin (all from Gibco; Thermo Fisher Scientific, Inc., Waltham, MA, USA) in a humidified $5 \% \mathrm{CO}_{2}$ at $37^{\circ} \mathrm{C}$.

ThemiR-139 mimics and negative control mimics (NC) were purchased from Shanghai GenePharma Co., Ltd. (Shanghai, China). HDGF-overexpressed plasmid (pcDNA3.1-HDGF) and the blank vector pcDNA3.1 (pcDNA3.1) were synthesized by the Chinese Academy of Sciences (Changchun, China). Transient transfections of the miRNA mimics or plasmid were conducted with Lipofectamine 2000 (Invitrogen; Thermo Fisher Scientific, Inc.) and OPTI-MEM reduced serum medium (Gibco; Thermo Fisher Scientific, Inc.), following the manufacturer's instructions.

Total RNA extraction and reverse transcription-quantitative polymerase chain reaction $(R T-q P C R)$. Total RNA and miRNAs were extracted from tissues or cells using TRIzol (Invitrogen; Thermo Fisher Scientific, Inc.) and mirVana miRNA isolation kit (Ambion; Thermo Fisher Scientific, Inc.), respectively. TaqMan microRNA assay (Applied Biosystems; Thermo Fisher Scientific, Inc.) was adopted to measure miR-139 expression. The reaction mixture contained $1.0 \mu \mathrm{l}$ TaqMan miRNA assay (20X), $10.0 \mu \mathrm{l}$ TaqMan 2X Universal PCR Master mix (Thermo Fisher Scientific, Inc.), $1.33 \mu 1$ cDNA, $1 \mu \mathrm{l}$ forward primer and $1 \mu 1$ reverse primer and $5.67 \mu \mathrm{l}$ double distilled water. For HDGF mRNA expression, reverse transcription was performed with PrimeScript ${ }^{\mathrm{TM}} \mathrm{RT}$ Master mix (Takara Bio, Inc., Otsu, Japan). The SYBR-Green PCR master mixture (Takara Bio, Inc.) was used to determine HDGF mRNA expression. The reaction system for qPCR consisted of $10 \mu 1$ SYBR-Green PCR Master mix, $2 \mu \mathrm{l}$ forward primer, $2 \mu \mathrm{l}$ reverse primer, $2 \mu \mathrm{l}$ cDNA and $4 \mu \mathrm{l}$ double distilled water. The thermocycler conditions were as follows: $95^{\circ} \mathrm{C}$ for $10 \mathrm{~min} ; 40$ cycles of $95^{\circ} \mathrm{C}$ for $15 \mathrm{sec}$ and $60^{\circ} \mathrm{C}$ for 1 min. RT-qPCR was performed in triplicate using ABI Prism 7500 Sequence Detection system (Applied Biosystems; Thermo Fisher Scientific, Inc.), the $2^{-\Delta \Delta C q}$ method was used to determine the relative gene expression (21). Primers are presented in Table I. U6 snRNA and GAPDH mRNA were used as endogenous controls for miR-139 and HDGF mRNA, respectively.
Table I. Primer sequences for reverse transcription-quantitative polymerase chain reaction.

\begin{tabular}{ll}
\hline Gene & \multicolumn{1}{c}{ Sequences $\left(5^{\prime} \rightarrow 3^{\prime}\right)$} \\
\hline miR-139 & \\
Forward & CGACGCGTCCCTCTTCCCATTCCTTC \\
Reverse & CCGGAATTCCGAGACCCACTGACACTATCT \\
U6 & \\
Forward & CTCGCTTCGGCAGCACATATACT \\
Reverse & ACGCTTCACGAATTTGCGTGTC \\
HDGF & \\
Forward & ATCAACAGCCAACAAATACC \\
Reverse & TTCTTATCACCGTCACCCT \\
GAPDH & \\
Forward & CATCACCATCTTCCAGGAGCG \\
Reverse & TGACCTTGCCCACAGCCTTG \\
\hline
\end{tabular}

MTT assay. The proliferative ability of EOC cells was determined using the 3-(4,5-dimethylthiazol-2-yl)-2,5-diphenyltetrazolium bromide (MTT) assay (Sigma-Aldrich; Merck Millipore, Darmstadt, Germany). Briefly, transfected cells were collected, counted and seeded in 96-well plates at a density of 2,000 cells/well. Cells were then incubated in a humidified $5 \% \mathrm{CO}_{2}$ at $37^{\circ} \mathrm{C}$ for continual $24-96 \mathrm{~h}$. The MTT assay was performed at specified time points, $20 \mu \mathrm{l}$ MTT solution $(5 \mathrm{mg} / \mathrm{ml})$ was added into each well and incubated at $37^{\circ} \mathrm{C}$ for $4 \mathrm{~h}$. Subsequently, the culture medium containing MTT solution was removed and replaced with $100 \mu 1$ DMSO (Sigma-Aldrich; Merck Millipore). Finally, the optical density at $490 \mathrm{~nm}$ (OD) was detected with a microplate reader (ELx800; Bio-Tek Instruments, Inc., Winooski, VT, USA).

Cell migration and invasion assay. The cell migration and invasion assay was performed in triplicate using Transwell chambers $(8-\mu \mathrm{m}$ pore size; EMD Millipore, Billerica, MA, USA) and Matrigel (BD Biosciences, San Jose, CA, USA)-coated Transwell chambers. In brief, transfected cells were collected at $48 \mathrm{~h}$ post-transfection, counted, and resuspended in FBS-free culture medium. A total of $4 \times 10^{4}$ cells in $100 \mu \mathrm{l} \mathrm{FBS-free} \mathrm{culture} \mathrm{medium} \mathrm{were} \mathrm{plated} \mathrm{into} \mathrm{the} \mathrm{upper}$ chamber, and $500 \mu \mathrm{l}$ culture medium containing $20 \%$ FBS was added to the lower chamber. Cells were incubated in a humidified $5 \% \mathrm{CO}_{2}$ at $37^{\circ} \mathrm{C}$ for $48 \mathrm{~h}$. Cells that remained on top of the filter were carefully removed with cotton swabs, and those that migrated or invaded through the membranes were fixed with 95\% methanol (Beyotime Institute of Biotechnology, Haimen, China), stained with $0.5 \%$ crystal violet (Beyotime Institute of Biotechnology) and photographed under a microscope (magnification, x200; Olympus Corporation, Tokyo, Japan).

Bioinformatics analysis. The target genes information of miR-139 were analyzed using TargetScan (www.targetscan.org) and miRanda (www.microrna.org).

Luciferase report assay. For the luciferase reporter assay, the wild type (Wt) 3'UTR and mutant (Mut) 3'UTR of 
HDGF into the pMIR-promotor vector was synthesized by Shanghai GenePharma Co., Ltd. HEK293T cells (ATCC) were seeded into 24-well plates at a density of 50-60\% influence. Subsequent to incubation overnight, cells were co-transfected with miR-139 mimics or NC, along with pMIR-HDGF-3'UTR Wt and pMIR-HDGF-3'UTR Mut using Lipofectamine 2000. Cells were then incubated in a humidified $5 \% \mathrm{CO}_{2}$ at $37^{\circ} \mathrm{C}$ for $48 \mathrm{~h}$, and luciferase activities were determined with the Dual-luciferase assay system (Promega Corporation, Madison, WI, USA), following to the manufacturer's instructions.

Western blotting. A total of $72 \mathrm{~h}$ post-transfection, cells were washed with ice-cold PBS (Gibco; Thermo Fisher Scientific, Inc.) three times and lysed in RIPA lysis buffer (Beyotime Institute of Biotechnology) supplemented with proteinase/phosphatase inhibitors (Thermo Fisher Scientific, Inc.). The concentration of total protein was detected by using a BCA assay kit (Beyotime Institute of Biotechnology). Equal amounts protein were subjected to $10 \%$ SDS polyacrylamide gel electrophoresis and transferred onto the polyvinylidene difluoride membranes (EMD Millipore). The membranes were then blocked with $3 \%$ skimmed milk in Tris-buffered saline $/ 0.1 \%$ Tween (TBST) at room temperature for $1 \mathrm{~h}$, and then incubated with primary antibodies at $4^{\circ} \mathrm{C}$ overnight. Subsequent to washing with TBST for three times, the membranes were incubated with goat anti-mouse horseradish peroxidase-conjugated secondary antibodies (cat. no. sc-2005; 1:5,000 dilution; Santa Cruz Biotechnology, Inc., Santa Cruz, CA, USA), and then visualized with enhanced chemiluminescence (Pierce; Thermo Fisher Scientific, Inc.). The primary antibodies used in the present study were mouse anti-human monoclonal HDGF (1:1,000 dilution; sc-271344; Santa Cruz Biotechnology, Inc.) and mouse anti-human monoclonal GAPDH (1:1,000 dilution; sc-51907; Santa Cruz Biotechnology, Inc.). GAPDH was used as an internal control for HDGF.

Statistical analysis. The results were presented as the mean \pm standard deviation. The differences between groups were compared using SPSS software, version 17 (SPSS, Inc., Chicago, IL, USA). Two-tailed $\mathrm{P}<0.05$ was considered to indicate a statistically significant difference.

\section{Results}

miR-139 expression in EOC tissues and cell lines. Firstly, miR-139 expression was assayed in EOC tissues and matched adjacent normal tissues using RT-qPCR. As presented in Fig. 1A, the expression level of miR-139 was significantly declined in EOC tissues compared with those in matched adjacent normal tissues $(\mathrm{P}<0.05)$. miR-139 expression in EOC cell lines (OVCAR3, CAOV3, SKOV3 and ES-2) in addition to the human NOEC was also determined. As predicted, miR-139 was significantly downregulated in EOC cell lines compared with NOEC (Fig. 1B; P<0.05).

miR-139 suppressed the proliferation and motility of EOC cells. The effects of miR-139 were further investigated on EOC carcinogenesis and progression. CAOV3 and SKOV3 were transfected with miR-139 mimics or NC. The upregulation of miR-139 on miR-139 mimics-transfected CAOV3 and
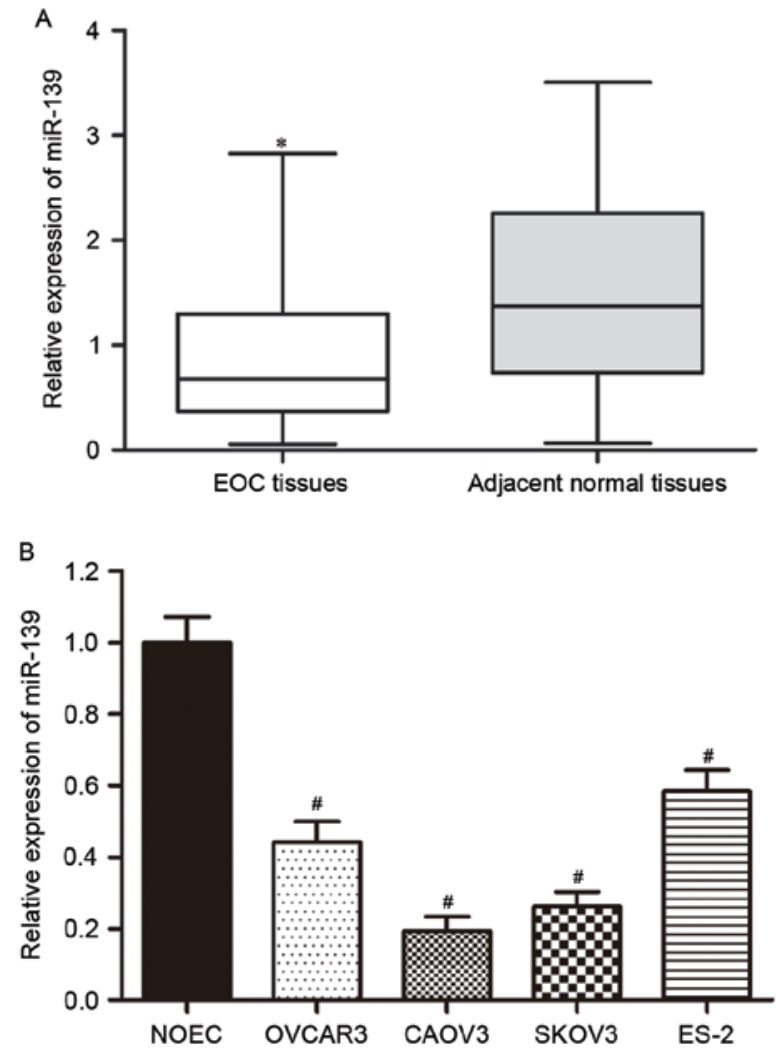

Figure 1. Reduced miR-139 expression in EOC tissues and cell lines. (A) Relative miR-139 expression in EOC tissues and matched adjacent normal tissues was measured using reverse transcription-quantitative polymerase chain reaction. ${ }^{\mathrm{P}}<0.05$ compared with adjacent normal tissues. (B) miR-139 expression level in EOC cell lines (OVCAR3, CAOV3, SKOV3, ES-2) and human NOEC. ${ }^{~ P}<0.05$ compared with NOEC. EOC, epithelial ovarian cancer; NOEC, normal ovarian epithelial cells.

SKOV3 was confirmed by RT-qPCR (Fig. 2A; P<0.05). MTT assay was performed to measure proliferation of miR-139 mimics-transfected CAOV3 and SKOV3 cells, and the results indicated that miR-139 overexpression inhibited proliferation of CAOV3 and SKOV3 cells (Fig. 2B; P<0.05). The effect of miR-139 on the motility of EOC cells was determined using cell migration and invasion assay. As presented in Fig. 2C, upregulation of miR-139 substantially suppressed migration and invasion capacities of CAOV3 and SKOV3 cells (Fig. 2C; $\mathrm{P}<0.05)$. These data suggested that miR-139 acted as a tumor suppressor in EOC.

$H D G F$ was a direct target gene of $m i R-139$. In order to explore the molecular mechanism of miR-139 in EOC, the predicted targets of miR-139 were analyzed using bioinformatics analysis with two publicly available databases (TargetScan and miRanda). As presented in Fig. 3A, the 3'UTR of HDGF contained potential binding sites of miR-139. To confirm HDGF as a direct target gene of miR-139, the luciferase reporter assay was performed. HEK293T cells were transfected with pMIR-HDGF-3'UTR Wt or pMIR-HDGF-3'UTR Mut, and miR-139 mimics or NC. As presented in Fig. 3B, results of luciferase reporter assay indicated that upregulation of miR-139 significantly reduced luciferase activities of pMIR-HDGF-3'UTR Wt $(\mathrm{P}<0.05)$, however not luciferase activities of pMIR-HDGF-3'UTR Mut. 

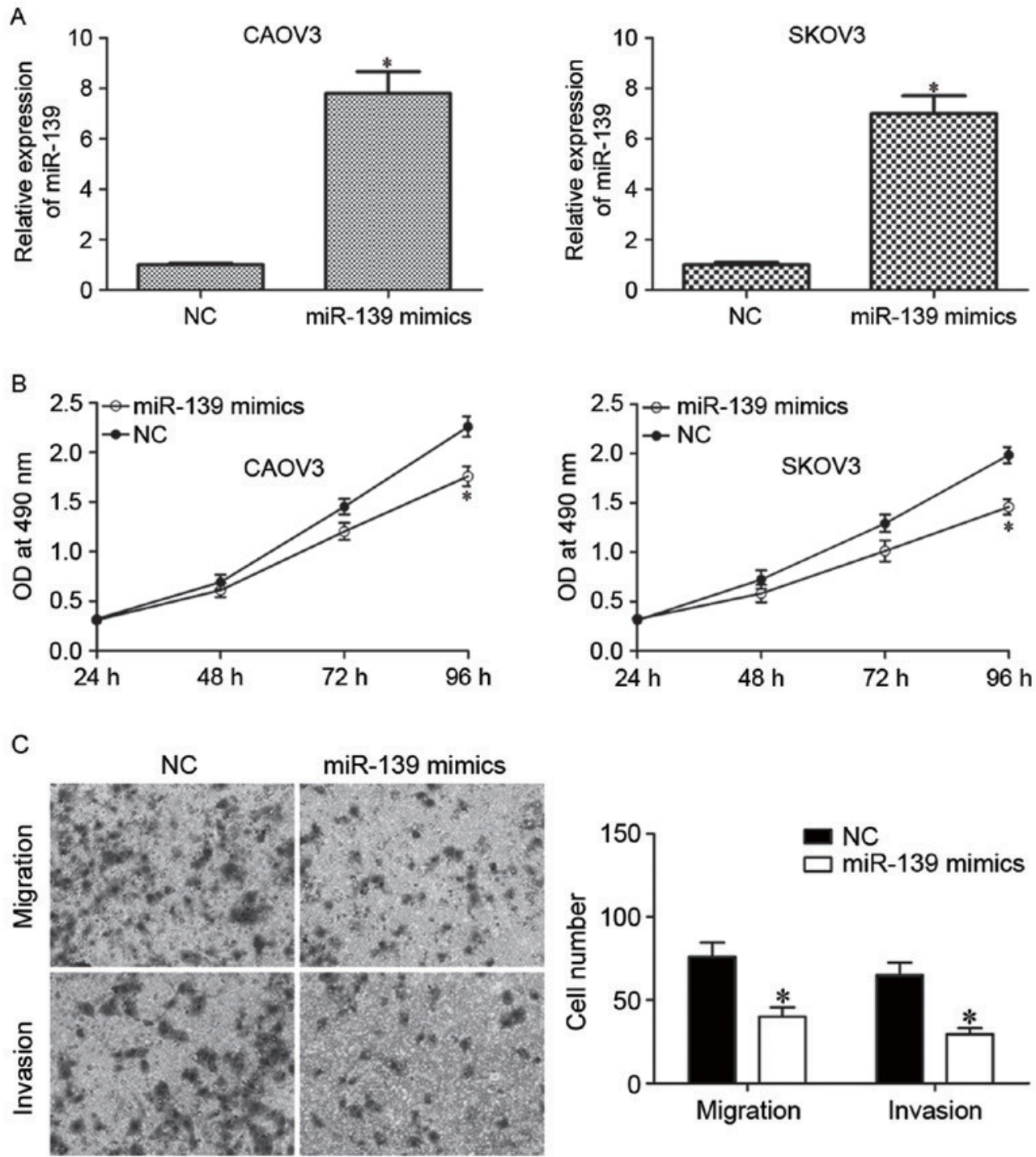

CAOV3
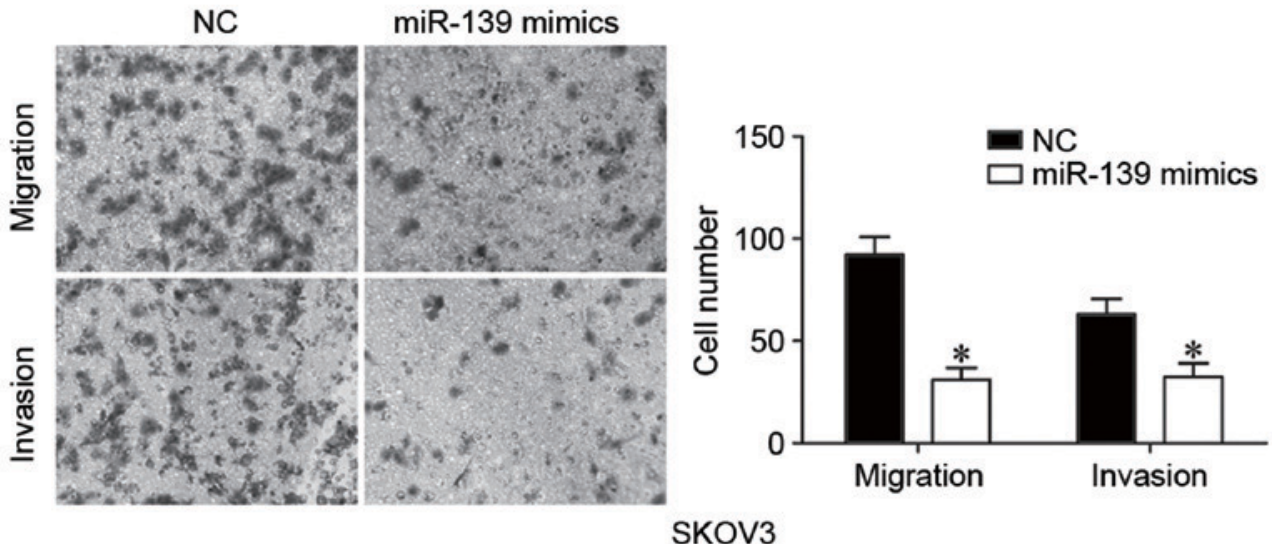

Figure 2. Upregulation of miR-139 inhibited growth and metastasis of EOC cells. (A) Following transfection with miR-139 mimics or NC, the miR-139 expression in CAOV3 and SKOV3 cells were detected using reverse transcription-quantitative polymerase chain reaction. ${ }^{*} \mathrm{P}<0.05$ compared with $\mathrm{NC}$. (B) Subsequent to miR-139 mimics or NC transfection, the cellular proliferation of CAOV3 and SKOV3 was assessed by the MTT assay. ${ }^{\text {P }}<0.05$ compared with NC. (C) Following miR-139 mimics or NC transfection, migration and invasion capacities of CAOV3 and SKOV3 cells were measured using the cell migration and invasion assay. " $\mathrm{P}<0.05$ compared with NC. miR, microRNA; EOC, epithelial ovarian cancer; NC, negative control; OD, optical density.

miR-139 negatively regulated HDGF expression in EOC cells. To investigate the regulation roles of miR-139 on HDGF expression, CAOV3 and SKOV3 cells were transfected with miR-139 mimics or NC, and measured HDGF expression by RT-qPCR and western blotting. The results of RT-qPCR indicated that restoration of miR-139 expression suppressed HDGF mRNA expression in CAOV3 and SKOV3 cells (Fig. 4A; $\mathrm{P}<0.05$ ). In addition, similar to the mRNA changes, 
A
HDGF 3' UTR

hsa-miR-139

HDGF 3' UTR Mut
Position 1265-1271

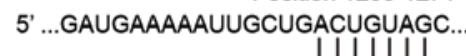

5 '...GAUGAAAAAUUGCUGUGACAUCC...
3' UGACCUCUGUGCACGUGACAUCU

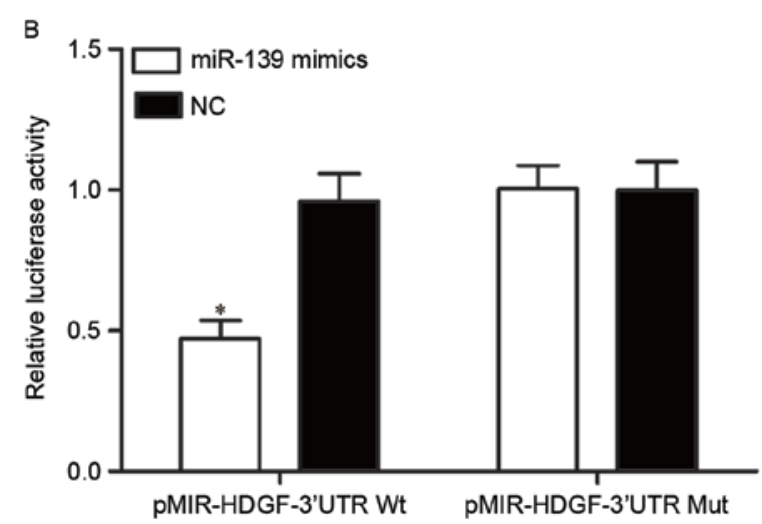

Figure 3. miR-139 directly targeted the 3'UTR of HDGF. (A) The predicted miR-139 target sequence in the 3'UTR of HDGF and a Mut containing altered nucleotides in the 3'UTR of HDGF. (B) HEK293T cells were transfected with pMIR-HDGF-3'UTR Wt or pMIR-HDGF-3'UTR Mut, together with miR-139 mimics or NC. Luciferase reporter assay was performed at $48 \mathrm{~h}$ post-transfection. " $\mathrm{P}<0.05$ compared with NC. miR, microRNA; UTR, untranslated region; HDGF, hepatoma-derived growth factor; Wt, wild-type; Mut, mutant.

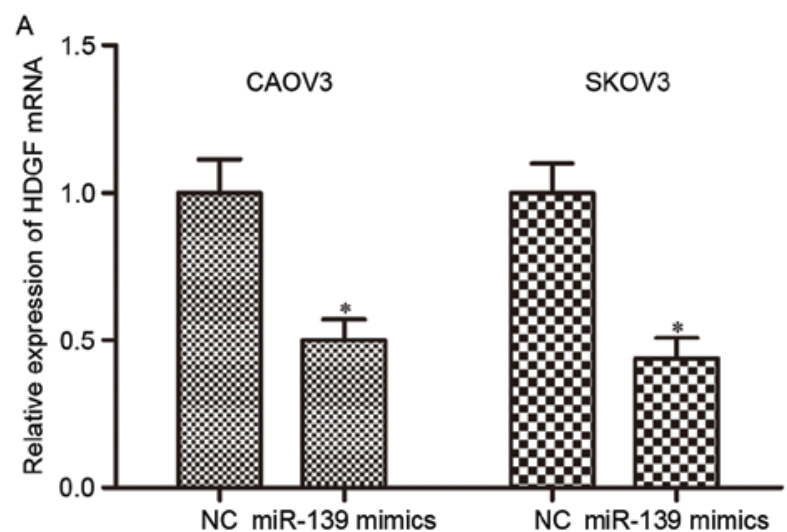

B

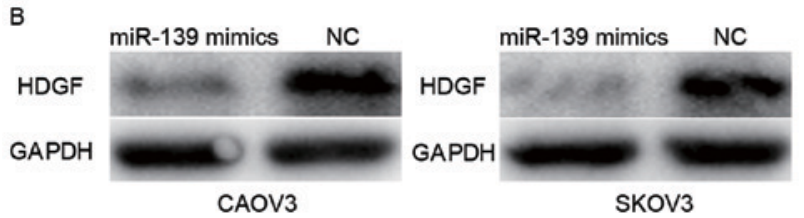

Figure 4. miR-139 reduced HDGF mRNA and protein expression level in EOC cells. (A) CAOV3 and SKOV3 cells were transfected with miR-139 mimics or NC. Expression of HDGF mRNA was determined using reverse transcription-quantitative polymerase chain reaction. ${ }^{\text {}} \mathrm{P}<0.05$ compared with NC. (B) HDGF protein levels were measured with western blotting. HDGF, hepatoma-derived growth factor; EOC, epithelial ovarian cancer; NC, negative control.

miR-139 overexpression additionally reduced HDGF protein expression level in CAOV3 and SKOV3 cells compared with NC groups (Fig. 4B; $\mathrm{P}<0.05$ ). These observations demonstrated that HDGF was a direct target gene of miR-139, and could negatively regulate HDGF expression through binding to its 3'UTR.
Upregulation of HDGF could rescue the inhibitory effects of miR-139 on EOC cells. HDGF was identified as a direct target gene of miR-139 in EOC. If these tumor suppressive roles of miR-139 on EOC were mediated by HDGF, HDGF overexpression could rescue these effects induced by miR-139. pcDNA3.1-HDGF or pcDNA3.1 was injected into CAOV3 and SKOV3 cells, and upregulation of HDGF was determined by RT-qPCR (Fig. 5A; P<0.05) and western blotting (Fig. 5B; $\mathrm{P}<0.05)$. Reintroduction of HDGF in miR-139 mimics-transfected CAOV3 and SKOV3 cells rescued the inhibitory effects on cell proliferation (Fig. 5C; $\mathrm{P}<0.05$ ) and motility (Fig. 5D and $\mathrm{E} ; \mathrm{P}<0.05$ ) induced by $\mathrm{miR}-139$ overexpression. These results further demonstrated that HDGF was a direct target of miR-139 in EOC.

\section{Discussion}

The dysregulation of miR-139 was a frequent event and it is involved in the carcinogenesis and progression of various kinds of human cancer. For example, in hepatocellular carcinoma, miR-139 expression was downregulated, and reduced miR-139 levels were correlated with clinicopathological features, including venous invasion, microsatellite formation, absence of tumor encapsulation and reduced differentiation (22). In colon cancer, expression levels of miR-139 were reduced in tumor tissues, and its low expression was associated with age. Furthermore, miR-139 underexpression was correlated with poor overall survival, particularly in patients with TNM stages I and II (23). In esophageal squamous cell carcinoma, reduced miR-139 expression was associated with lymph node metastases (24). The downregulation of miR-139 was also observed to correlate with gastric cancer (25), parathyroid carcinoma (26), and basal cell carcinoma (27). However, the expression pattern of miR-139 in EOC was not investigated. In the present study, it was observed that miR-139 was significantly downregulated in EOC tissues and cell lines using RT-qPCR. These observations suggested that miR-139 may serve important roles in cancer.

miR-139 has been subject to various studies and serves significant roles in numerous biological functions. In hepatocellular carcinoma, upregulation of miR-139 clearly attenuated cell motility in vitro and the incidence and severity of lung metastasis from orthotopic liver tumors in vivo through negative regulation of rho associated coiled-coil containing protein kinase 2 (22). Gu et al (28) reported that miR-139 overexpression suppressed hepatocellular carcinoma cells growth, migration, invasion and enhanced apoptosis via the WNT/TCF-4 pathway. In glioma, miR-139 inhibited cell proliferation and invasion both in vitro and in vivo through directly targeting IGF-1R, AMY-1 and PGC-1 $\beta$ (29). In addition, restoration of miR-139 repressed glioma cells migration and invasion by targeting ZEB1 and ZEB2 (30), and improved temozolomide-induced apoptosis via blockade of MCl-1 (31). Ren et al (32) demonstrated that miR-139 decreased cell growth and induced cell apoptosis via the protein kinase B signaling pathway. Zhang et al (33) observed that miR-139 overexpression inhibited breast cancer cell growth, motility, enhanced cell apoptosis, caused cell cycle arrest in $\mathrm{S}$ phase and improved chemosensitivity to docetaxel by targeting Notch1. However, the roles of miR-139 on EOC cells remained 
A

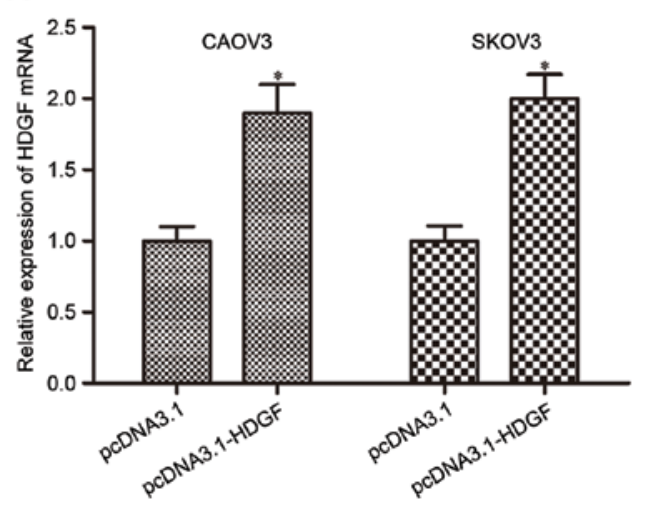

C

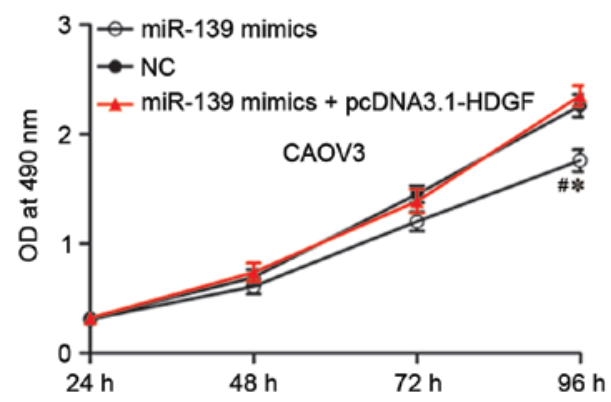

B

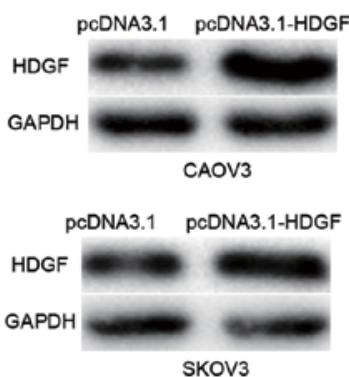

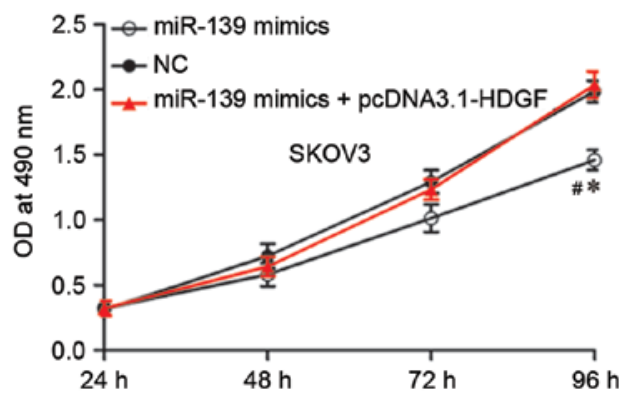

D

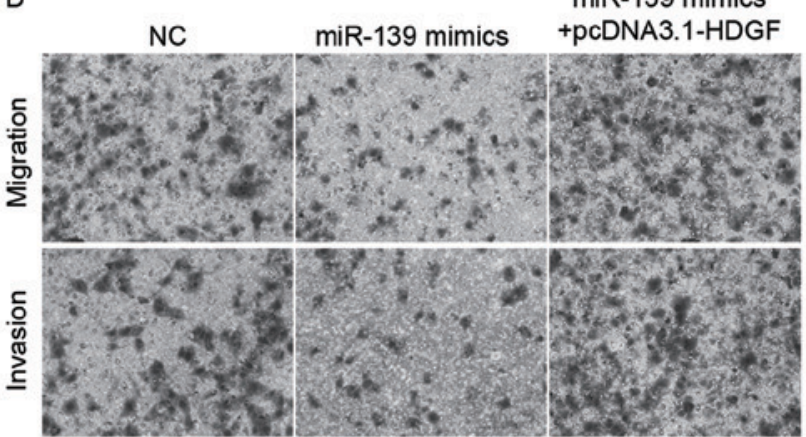

E
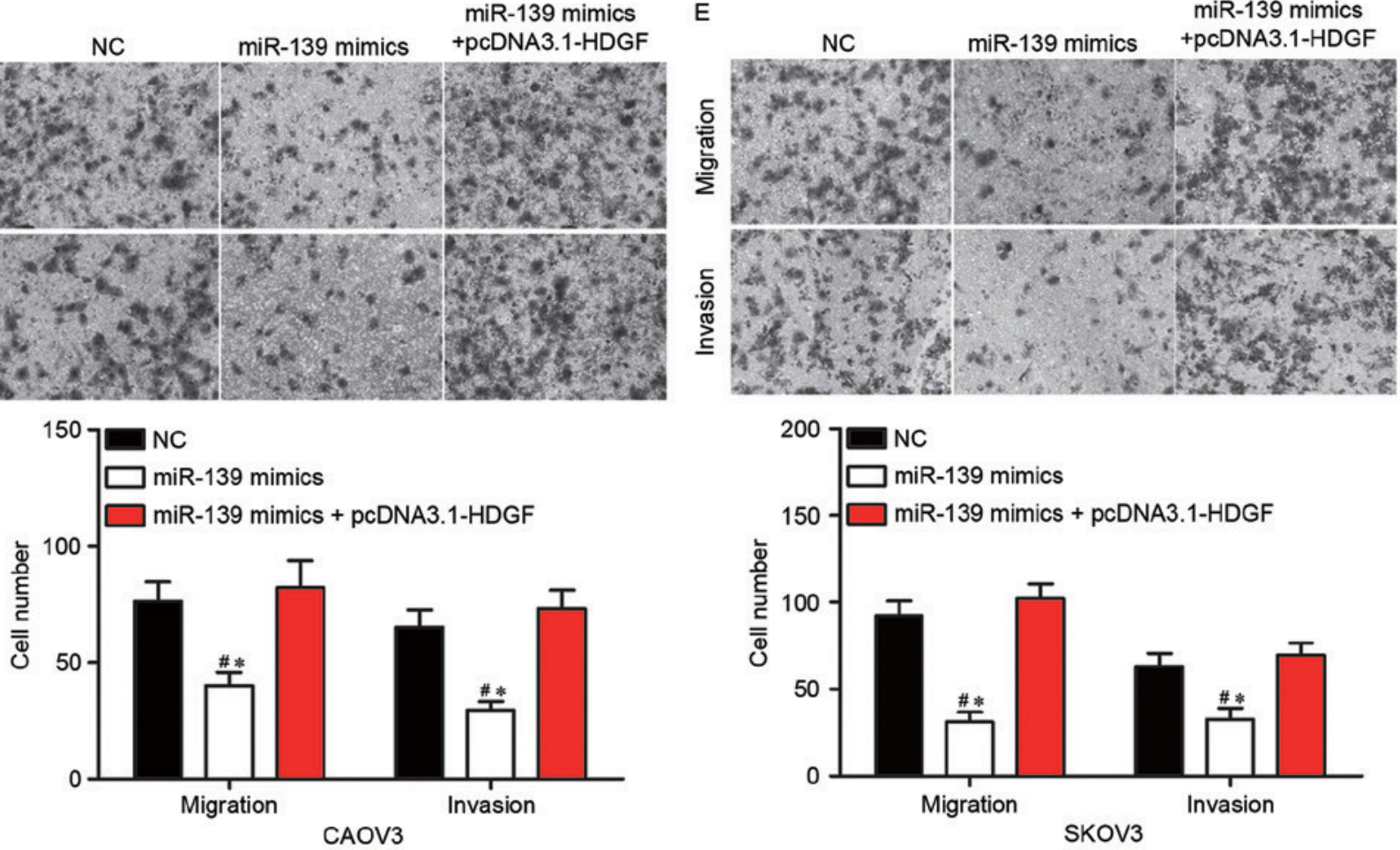

Figure 5. HDGF overexpression rescued the inhibitory effects of miR-139 on EOC cells. (A) Following transfection with pcDNA3.1-HDGF or pcDNA3.1, HDGF mRNA expression in CAOV3 and SKOV3 cells were detected using reverse transcription-quantitative polymerase chain reaction. ${ }^{*} \mathrm{P}<0.05$ compared with pcDNA3.1. (B) HDGF protein levels in CAOV3 and SKOV3 cells transfected with pcDNA3.1-HDGF or pcDNA3.1 were detected with western blotting. (C) Following transfection with pcDNA3.1-HDGF or pcDNA3.1, an MTT assay was performed to evaluate proliferation in CAOV3 and SKOV3 cells. "P $<0.05$ compared with NC, ${ }^{\text {"}} \mathrm{P}<0.05$ compared with miR-139 mimics+pcDNA3.1-HDGF. Following pcDNA3.1-HDGF or pcDNA3.1 transfection, cell migration and invasion assays were performed to assess effects of HDGF overexpression on motility of (D) CAOV3 and (E) SKOV3 cells. "P $<0.05$ compared with NC; "P $<0.05$ compared with miR-139 mimics+pcDNA3.1-HDGF. HDGF, hepatoma-derived growth factor; miR, microRNA; EOC, epithelial ovarian cancer; NC, negative control.

to be investigated. The current study observed that ectopic of miR-139 expression significantly inhibited proliferation, migration and invasion of EOC cells. These results suggested that miR-139 acted as a tumor suppressor in cancer, and could be investigated as a therapeutic target for the therapy of these types of cancer.

Regarding the molecular mechanism underlying the regulation effect of miRNA on cancers, it is crucial to explore 
their target genes. Firstly, bioinformatics analysis indicated the presence of miR-139 binding site on the 3'UTR of HDGF. In addition, luciferase reporter assays indicated that miR-139 overexpression decreased the luciferase activities of Wt 3'UTR of HDGF, whereas there was no effect on the Mut 3'UTR of HDGF, suggesting that HDGF was a direct target gene of miR-139. In addition, results of RT-qPCR and western blotting indicated that enforced miR-139 expression suppressed HDGF expression at both mRNA and protein levels in EOC cells. Finally, upregulation of HDGF in miR-139 mimics-transfected cells could rescue the tumor suppressive roles induced by miR-139 overexpression on cell proliferation, migration and invasion, further demonstrating that miR-139 inhibited EOC cell proliferation, migration and invasion through directly targeting HDGF.

HDGF, located on chromosome 1, region q21-q23 (34), is a heparin-binding growth factor and firstly purified from culture medium conditioned with hepatoma-cell line $\mathrm{HuH7}$ (35). In EOC, HDGF was significantly upregulated in tumor tissues and higher expression of HDGF was correlated with lymphatic metastasis. In addition, patients with EOC with higher HDGF levels had a poorer five-year overall survival rate, and multivariate analysis identified HDGF as an independent prognostic factor for patients with EOC (36). Therefore, regarding its cancer-associated functions, HDGF is worthwhile to be investigated as a novel therapeutic target in EOC. Increasing studies indicated that HDGF may be regulated by multiple miRNAs in various types of cancer, including miR-610 in colorectal cancer (37), miR-497 in prostate cancer (38), miR-195 in non-small cell lung cancer (39), miR-141 in gastric cancer (40), and miR-214 in hepatocellular carcinoma (41). In the present study, to the best of our knowledge for the first time, it was observed that HDGF may be negatively regulated by miR-139 in EOC, and therefore to inhibit cell growth and metastasis. Collectively, miR-139 could be investigated as a targeted therapy to against HDGF and to block EOC rapidly growth and metastasis.

In conclusion, the present study indicated that miR-139 exhibited tumor suppressive roles against EOC through directly targeting HDGF. This newly identified miR-139/HDGF association provided potential novel therapeutic targets for patients with EOC.

\section{References}

1. Ferlay J, Soerjomataram I, Dikshit R, Eser S, Mathers C, Rebelo M, Parkin DM, Forman D and Bray F: Cancer incidence and mortality worldwide: Sources, methods and major patterns in GLOBOCAN 2012. Int J Cancer 136: E359-E386, 2015.

2. Suh DH, Kim JW, Kim K, Kim HJ and Lee KH: Major clinical research advances in gynecologic cancer in 2012. J Gynecol Oncol 24: 66-82, 2013.

3. Maldonado L and Hoque MO: Epigenomics and ovarian carcinoma. Biomark Med 4: 543-570, 2010.

4. Dong R, Liu X, Zhang Q, Jiang Z, Li Y, Wei Y, Li Y, Yang Q, Liu J, Wei JJ, et al: miR-145 inhibits tumor growth and metastasis by targeting metadherin in high-grade serous ovarian carcinoma. Oncotarget 5: 10816-10829, 2014.

5. Yiwei T, Hua H, Hui G, Mao M and Xiang L: HOTAIR Interacting with MAPK1 regulates ovarian cancer skov3 cell proliferation, migration, and invasion. Med Sci Monit 21: 1856-1863, 2015.

6. Dwivedi SK, Mustafi SB, Mangala LS, Jiang D, Pradeep S, Rodriguez-Aguayo C, Ling H, Ivan C, Mukherjee P, Calin GA, et al: Therapeutic evaluation of microRNA-15a and microRNA-16 in ovarian cancer. Oncotarget 7: 15093-15104, 2016.
7. Chen X, Dong C, Law PT, Chan MT, Su Z, Wang S, Wu WK and $\mathrm{Xu} \mathrm{H}$ : MicroRNA-145 targets TRIM2 and exerts tumor-suppressing functions in epithelial ovarian cancer. Gynecol Oncol 139: 513-519, 2015.

8. Luo J, Zhou J, Cheng Q, Zhou C and Ding Z: Role of microRNA-133a in epithelial ovarian cancer pathogenesis and progression. Oncol Lett 7: 1043-1048, 2014.

9. Bartel DP: MicroRNAs: Genomics, biogenesis, mechanism, and function. Cell 116: 281-297, 2004.

10. Esquela-Kerscher A and Slack FJ: Oncomirs-microRNAs with a role in cancer. Nat Rev Cancer 6: 259-269, 2006.

11. Schmiedel JM, Klemm SL, Zheng Y, Sahay A, Bluthgen N, Marks DS and van Oudenaarden A: Gene expression. MicroRNA control of protein expression noise. Science 348: 128-132, 2015.

12. Ebert MS and Sharp PA: Roles for microRNAs in conferring robustness to biological processes. Cell 149: 515-524, 2012.

13. Bartel DP: MicroRNAs: Target recognition and regulatory functions. Cell 136: 215-233, 2009.

14. Friedman RC, Farh KK, Burge CB and Bartel DP: Most mammalian mRNAs are conserved targets of microRNAs. Genome Res 19: 92-105, 2009.

15. Sasaki H, Yoshiike M, Nozawa S, Usuba W, Katsuoka Y, Aida K, Kitajima K, Kudo H, Hoshikawa M, Yoshioka Y, et al: Expression level of urinary microRNA-146a-5p is increased in patients with bladder cancer and decreased in those after transurethral resection. Clin Genitourin Cancer 14: e493-e499, 2016.

16. Li Y, Jin L, Chen D, Liu J, Su Z, Yang S, Gui Y, Mao X, Nie G and Lai Y: Tumor suppressive miR-196a is associated with cellular migration, proliferation and apoptosis in renal cell carcinoma. Mol Med Rep 14: 560-566, 2016.

17. Chen Z, Liu H, Jin W, Ding Z, Zheng S and Yu Y: Tissue microRNA-21 expression predicted recurrence and poor survival in patients with colorectal cancer-a meta-analysis. Onco Targets Ther 9: 2615-2624, 2016.

18. Tao S, Liu YB, Zhou ZW, Lian B, Li H, Li JP and Zhou SF: miR-3646 promotes cell proliferation, migration, and invasion via regulating G2/M transition in human breast cancer cells. Am J Transl Res 8: 1659-1677, 2016.

19. Wang N, Wang Q, Shen D, Sun X, Cao X and Wu D: Downregulation of microRNA-122 promotes proliferation, migration, and invasion of human hepatocellular carcinoma cells by activating epithelial-mesenchymal transition. Onco Targets Ther 9: 2035-2047, 2016.

20. Chen H, Zhang L, Zhang L, Du J, Wang $\mathrm{H}$ and Wang B: MicroRNA-183 correlates cancer prognosis, regulates cancer proliferation and bufalin sensitivity in epithelial ovarian caner. Am J Transl Res 8: 1748-1755, 2016.

21. Livak KJ and Schmittgen TD: Analysis of relative gene expression data using real-time quantitative PCR and the 2(-Delta Delta C(T)) Method. Methods 25: 402-408, 2001

22. Wong CC, Wong CM, Tung EK, Au SL, Lee JM, Poon RT, Man K and Ng IO: The microRNA miR-139 suppresses metastasis and progression of hepatocellular carcinoma by down-regulating Rho-kinase 2. Gastroenterology 140: 322-331, 2011.

23. Liu X, Duan B, Dong Y, He C, Zhou H, Sheng H, Gao H and Zhang X: MicroRNA-139-3p indicates a poor prognosis of colon cancer. Int J Clin Exp Pathol 7: 8046-8052, 2014.

24. Liu R, Yang M, Meng Y, Liao J, Sheng J, Pu Y, Yin L and Kim SJ: Tumor-suppressive function of miR-139-5p in esophageal squamous cell carcinoma. PLoS One 8: e77068, 2013

25. Guo J, Miao Y, Xiao B, Huan R, Jiang Z, Meng D and Wang Y: Differential expression of microRNA species in human gastric cancer versus non-tumorous tissues. J Gastroenterol Hepatol 24: 652-657, 2009.

26. Corbetta S, Vaira V, Guarnieri V, Scillitani A, Eller-Vainicher C, Ferrero S, Vicentini L, Chiodini I, Bisceglia M, Beck-Peccoz P, et al: Differential expression of microRNAs in human parathyroid carcinomas compared with normal parathyroid tissue. Endocr Relat Cancer 17: 135-146, 2010.

27. Sand M, Skrygan M, Sand D, Georgas D, Hahn SA, Gambichler T, Altmeyer P and Bechara FG: Expression of microRNAs in basal cell carcinoma. Br J Dermatol 167: 847-855, 2012.

28. Gu W, Li X and Wang J: miR-139 regulates the proliferation and invasion of hepatocellular carcinoma through the WNT/TCF-4 pathway. Oncol Rep 31: 397-404, 2014.

29. Wang H, Yan X, Ji LY, Ji XT, Wang P, Guo SW and Li SZ: miR-139 functions as an antioncomir to repress glioma progression through targeting IGF-1 R, AMY-1, and PGC-1 $\beta$. Technol Cancer Res Treat: Feb 10, 2016 (Epub ahead of print). 
30. Yue S, Wang L, Zhang H, Min Y, Lou Y, Sun H, Jiang Y, Zhang W, Liang A, Guo Y, et al: miR-139-5p suppresses cancer cell migration and invasion through targeting ZEB1 and ZEB2 in GBM. Tumour Biol 36: 6741-6749, 2015.

31. Li RY, Chen LC, Zhang HY, Du WZ, Feng Y, Wang HB, Wen JQ Liu X, Li XF, Sun Y, et al: MiR-139 inhibits Mcl-1 expression and potentiates TMZ-induced apoptosis in glioma. CNS Neurose Ther 19: 477-483, 2013

32. Ren Y, Zhu H, Chi C, Yang F and Xu X: MiRNA-139 regulates oral cancer Tca8113 cells apoptosis through Akt signaling pathway. Int J Clin Exp Pathol 8: 4588-4594, 2015.

33. Zhang HD, Sun DW, Mao L, Zhang J, Jiang LH, Li J, Wu Y, Ji H, Chen W, Wang J, et al: MiR-139-5p inhibits the biological function of breast cancer cells by targeting Notch1 and mediates chemosensitivity to docetaxel. Biochem Biophys Res Commun 465: 702-713, 2015.

34. Bao C, Wang J, Ma W, Wang X and Cheng Y: HDGF: A novel jack-of-all-trades in cancer. Future Oncol 10: 2675-2685, 2014

35. Huang JS, Chao CC, Su TL, Yeh SH, Chen DS, Chen CT, Chen PJ and Jou YS: Diverse cellular transformation capability of overexpressed genes in human hepatocellular carcinoma. Biochem Biophys Res Commun 315: 950-958, 2004.
36. Liu XJ,Liu WL, Yang FM, Yang XQ and Lu XF: Hepatoma-derived grow th factor predicts unfavorable prognosis of epithelial ovarian cancer. Onco Targets Ther 8: 2101-2109, 2015.

37. Sun B, Gu X, Chen Z and Xiang J: MiR-610 inhibits cell proliferation and invasion in colorectal cancer by repressing hepatoma-derived growth factor. Am J Cancer Res 5: 3635-3644, 2015.

38. Wu D, Niu X, Pan H, Zhang Z, Zhou Y, Qu P and Zhou J: MicroRNA-497 targets hepatoma-derived growth factor and suppresses human prostate cancer cell motility. Mol Med Rep 13: 2287-2292, 2016.

39. Guo H, Li W, Zheng T and Liu Z: MiR-195 targets HDGF to inhibit proliferation and invasion of NSCLC cells. Tumour Biol 35: 8861-8866, 2014.

40. Chen B, Huang T, Jiang J, Lv L, Li H and Xia S: miR-141 suppresses proliferation and motility of gastric cancer cells by targeting HDGF. Mol Cell Biochem 388: 211-218, 2014.

41. Shih TC, Tien YJ, Wen CJ, Yeh TS, Yu MC, Huang CH, Lee YS, Yen TC and Hsieh SY: MicroRNA-214 downregulation contributes to tumor angiogenesis by inducing secretion of the hepatoma-derived growth factor in human hepatoma. J Hepatol 57: 584-591, 2012. 